\title{
Inspection and selection of representations
}

\author{
Daniel Raggi ${ }^{1}$, Aaron Stockdill ${ }^{1}$, Mateja Jamnik ${ }^{1}$, \\ Grecia Garcia Garcia ${ }^{2}$, Holly E. A. Sutherland ${ }^{2}$, and Peter C.-H. Cheng ${ }^{2}$ \\ 1 University of Cambridge, UK \\ \{daniel.raggi, aaron.stockdill, mateja.jamnik\}@cl.cam.ac.uk \\ ${ }^{2}$ University of Sussex, UK \\ $\{$ g.garcia-garcia, h.sutherland, p.c.h.cheng\}esussex.ac.uk
}

\begin{abstract}
We present a novel framework for inspecting representations and encoding their formal properties. This enables us to assess and compare the informational and cognitive value of different representations for reasoning. The purpose of our framework is to automate the process of representation selection, taking into account the candidate representation's match to the problem at hand and to the user's specific cognitive profile. This requires a language for talking about representations, and methods for analysing their relative advantages. This foundational work is first to devise a computational end-to-end framework where problems, representations, and user's profiles can be described and analysed. As AI systems become ubiquitous, it is important for them to be more compatible with human reasoning, and our framework enables just that.
\end{abstract}

Keywords: Representation in reasoning, heterogeneous reasoning, representation selection, representational system.

\section{Introduction}

The aim of this work is to contribute to the development of AI systems which, similarly to human experts, can pick effective representations for the task at hand.

The effectiveness of a representation depends on its purpose. One kind of representation may be more useful for problem solving, while another may facilitate learning, and some may be more suitable for school children, while another may be useful for the working professional. Thus, for a system to select representations intelligently, it needs to take into account both the formal (structure and information) and cognitive (user and task) aspects of representations.

This is interdisciplinary foundational work bringing together artificial intelligence, computer science and cognitive science to devise a framework (the language and methods) for analysing and encoding the properties of representations. In this paper, we focus on the formal properties of representations, and the analysis involved in finding a matching representation for the given problem amongst a variety of candidate representational systems. This analysis is done in a purely structural and informational manner (i.e., without taking into account the user and task). As we will argue, the formal properties are a foundational layer upon which the cognitive properties of representations depend. The details of the analysis and encoding of cognitive properties, which bring the user profile into the picture, are described elsewhere [11]. 
Furthermore, we present a proof-of-concept application of our framework, where multiple representational systems are automatically evaluated relative to a problem, by a measure that estimates how likely each representational system is to contain all the ingredients for finding a solution.

Automating the process of analysing and evaluating representations would lead to a new generation of interactive computer systems which adapt intelligently to the user. The automatic selection of effective representations could have applications ranging from intelligent tutoring systems, to systems that aid the working scientist, to fully automated problem-solvers.

\section{The role of representation in reasoning}

The advantages of particular representations over others have been extensively discussed $[5,6,26]$. Furthermore, there are evident cognitive benefits of multiple representations over single representations [1].

Various formalisations of specific reasoning systems using a single kind of representation have been implemented, including first order [16, 17], higher order [12, 22], diagrammatic [14, 28, 29], among many others. A few heterogeneous reasoning systems that integrate multiple representations have also been built [2, 27], as well as some tools for re-representing problems and knowledge across and within systems $[13,20,24]$. Many systems designed for numerical, algebraic, and geometric computing include tools for representing data in various ways (graphs, plots, figures, diagrams, etc.) $[15,19,23,25]$.

Moreover, in the rapidly-advancing areas of AI (i.e., machine learning), the role that representation plays has been recognised as crucial to the effective processing of data, and representation learning has become a rich area of research [3]. However, the gap between the actual computations and the user's understanding seems to be increasing as the tools perform more effectively under more autonomous (and obscure) conditions. To reduce this gap, it is necessary to understand what makes representations better or worse for humans.

Some work has been done to understand the qualities of representations [4, 7]. However, to our knowledge, there is no integration of this knowledge into a framework where representational systems can be analysed, evaluated, and selected computationally, where the task and user can be taken into account. In this work we set some foundations to approach the automation of representation analysis and selection.

\subsection{An example}

To illustrate the variety and efficacy of representations for reasoning, we first present a problem in probability with three example solutions.

Problem (Birds). One quarter of all animals are birds. Two thirds of all birds can fly. Half of all flying animals are birds. Birds have feathers. If $X$ is an animal, what is the probability that it's not a bird and it cannot fly? 
Solution (Bayesian): Let the sample space be the set of animals. Let $b$ represent birds, $f$ flying animals, and $\operatorname{Pr}$ the probability function. Then, the problem can be reformulated, in the language of conditional probability, as follows:

Assume: $\quad \operatorname{Pr}(b)=\frac{1}{4}, \operatorname{Pr}(f \mid b)=\frac{2}{3}, \operatorname{Pr}(b \mid f)=\frac{1}{2}$.

Calculate: $\operatorname{Pr}(\bar{b} \cap \bar{f})$

To solve this, we start by noting the following facts:

$$
\begin{aligned}
\operatorname{Pr}(\bar{b}) & =\operatorname{Pr}(\bar{b} \cap \bar{f})+\operatorname{Pr}(\bar{b} \cap f) \\
\operatorname{Pr}(f) & =\operatorname{Pr}(b \cap f)+\operatorname{Pr}(\bar{b} \cap f) \\
\operatorname{Pr}(\bar{b} \cap f) & =\operatorname{Pr}(\bar{b} \mid f) \operatorname{Pr}(f)=\frac{1}{2} \operatorname{Pr}(f) .
\end{aligned}
$$

From (2) and (3) we can show that $\operatorname{Pr}(\bar{b} \cap f)=\frac{1}{2} \operatorname{Pr}(b \cap f)+\frac{1}{2} \operatorname{Pr}(\bar{b} \cap f)$, from which we obtain

$$
\operatorname{Pr}(\bar{b} \cap f)=\operatorname{Pr}(b \cap f) .
$$

Thus, we have the following:

$$
\begin{aligned}
\operatorname{Pr}(\bar{b} \cap \bar{f}) & =\operatorname{Pr}(\bar{b})-\operatorname{Pr}(\bar{b} \cap f) & & \text { from (1) } \\
& =\operatorname{Pr}(\bar{b})-\operatorname{Pr}(b \cap f) & & \text { from (4) } \\
& =(1-\operatorname{Pr}(b))-\operatorname{Pr}(f \mid b) \operatorname{Pr}(b) & & \text { from probability axioms } \\
& =\frac{3}{4}-\left(\frac{2}{3}\right)\left(\frac{1}{4}\right)=\frac{7}{12} . & & \text { from assumptions }
\end{aligned}
$$

Solution (Geometric): In the figure below, the unit square represents the set of animals. The regions representing birds and flying animals are drawn according to the assumptions.

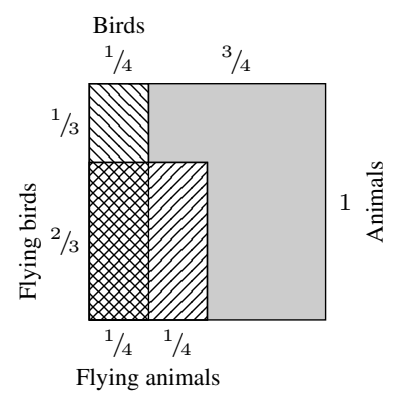

It is clear that the shaded region (non-flying, non-bird animals) has an area of $\frac{3}{4}-\left(\frac{2}{3}\right)\left(\frac{1}{4}\right)=\frac{7}{12}$. 
Solution (Contingency): We start by building a table from the two relevant classes (birds and flying animals) and fill in the values that we are given. Next, the table is filled in gradually, according to simple tabular constraints.

\begin{tabular}{|l|l|l|l|}
\hline & birds & non-birds & total \\
\hline flying & $(2 / 3)(1 / 4)$ & $(2 / 3)(1 / 4)$ & \\
\hline non-flying & & $3 / 4-(2 / 3)(1 / 4)$ & \\
\hline total & $1 / 4$ & $3 / 4$ & 1 \\
\hline
\end{tabular}

The rules used to fill the rest of the table are either transferred from the problem's statement (e.g., half of all flying animals are birds means that top cells must have the same values), or from probability theorems (e.g., the total probability is the sum of the parts means that the total of each column/row must be the sum of the cells in the corresponding column/row). It's worth noting that to the experienced user of contingency tables, the constraints corresponding to probability theorems do not need to be explicitly invoked from probability theory, but simply recalled as tabular/arithmetic manipulations. Thus, we reach the solution when we fill in the shaded cell.

Note that there may be many solutions using the same representational system (e.g., many Bayesian solutions, many Geometric solutions, etc.). In the next section we present the framework for analysing these representations.

\section{Representing representations}

Representational systems are diverse, and their designs and specifications are heterogeneous. To do a comparative analysis, we need a common framework that captures the properties that make up each representation.

Our framework is based around three concepts: representational system (RS), problem $(\mathrm{Q})$, and correspondence. In the following subsections we suggest a concrete method for inspecting and encoding their properties. The result is a set of machine-readable tables suitable for further analysis.

Table construction currently needs to be done by a human analyst, but the purpose of such tables is that they can be processed automatically to yield a representation recommendation.

\subsection{Representational system}

We view representational systems as consisting of two layers implemented over a medium: a grammatical layer, and an inferential layer. Broadly speaking, the grammatical layer distinguishes the sensible from the nonsensical, and the inferential layer distinguishes the valid from the invalid.

The table of an RS is a structured description of the RS. It is a collection of the most relevant properties of the RS. We encode these properties as pairs $(k, v)$, where $k$ is the property kind and $v$ is the value. For example, if an RS table has the entry (rigorous, TRUE) this means that the RS has the property of being rigorous; and if it has (token, + ) this means that the token ' + ' is part of the symbols used in the RS (for examples of RS tables, see Tables 1 and 2). 
Grammar. The building blocks of an RS are called tokens. These are used to construct expressions. The grammar of an RS allows us to distinguish between admissible and inadmissible expressions. Although grammars can be constructed in various ways (e.g., by production rules [8], or by some underlying type theory [10]), our framework is agnostic to the specific construction, but embraces type theory as a generic descriptive tool. Thus, every token, and every admissible expression will be assumed to have a type. However, to keep our framework flexible, we make no other assumptions about the specific type theory that we use.

We encode the property of grammatical complexity (g-complexity in Tables 1 and 2) using Chomsky hierarchies, which are linked to well-known classes of computational complexity and expressiveness [9].

Inference. The medium on which an RS is implemented (e.g., a pen and paper, or a computer interface) can be manipulated by some agent. Thus, we need a concept of state. Broadly speaking, a state refers to the conditions of a medium at some moment in time. Manipulations are changes of state.

The inferential layer of an RS determines how the grammatical expressions of an RS can be manipulated validly. Borrowing from the field of computer-assisted theorem proving, we refer to valid manipulations as tactics. Moreover, tactics are often parametric on knowledge. The knowledge encoded in an RS can be represented as a collection of

Table 1. A section of the Bayesian RS table.

\begin{tabular}{|ll|}
\hline kind & value \\
\hline types & real, event \\
\hline tokens & $\Omega, \emptyset, 0,1,=,+,-, *, \div, \cup, \cap, \backslash,-, \mathrm{Pr}, \mid$ \\
\hline g-complexity & type-2 \\
\hline facts & $\begin{array}{l}\text { Bayes' theorem, law of total probability, non-negative probabil- } \\
\text { ity, unit-measure, sigma-additivity, commutativity ... }\end{array}$ \\
\hline tactics & rewrite, arithmetic calculation \\
\hline i-complexity & 3 \\
\hline rigorous & TRUE \\
\hline
\end{tabular}

Table 2. A section of the Geometric RS table.

\begin{tabular}{|l|l|}
\hline kind & value \\
\hline types & point, segment, region, real, string \\
\hline tokens & $\begin{array}{l}\text { \$point, \$segment (the prefix \$ denotes a label for an actually } \\
\text { pictorial symbol) }\end{array}$ \\
\hline g-complexity & type-3 \\
\hline facts & $\begin{array}{l}\text { scale-independence of ratio, non-negative area, area- } \\
\text { additivity,... }\end{array}$ \\
\hline tactics & draw point, draw segment, delete, join, compare sizes \\
\hline i-complexity & 2 \\
\hline rigorous & TRUE \\
\hline
\end{tabular}


facts. These can be formulae (e.g., in Bayesian system $\operatorname{Pr}(x)=\operatorname{Pr}(x \wedge y)+\operatorname{Pr}(x \wedge \neg y)$ ), extrinsically-imposed constraints (e.g., in contingency tables the last value of a row must be the sum of the values of the row), or intrinsic constraints (e.g., the area of a region is the sum of the areas of its parts). Thus, tactics and facts are the main constituent properties of an RS.

As with grammatical complexity, inferential complexity (i-complexity in Tables 1 and 2) can be measured according to known standards. The partial order induced by injective embeddings of theories within one another could be a basis by which to compare systems, but a radical simplification by flattening into 5 classes turns out to be enough for a rough assessment of complexity. We propose the following levels (with a known system for reference): 1 (propositional logic), 2 (decidable fractions of arithmetic/set theory), 3 (Peano arithmetic), 4 (constructive set theory), and 5 (Zermelo-Fraenkel).

Furthermore, we include the property of rigour to describe whether the calculations/proofs are guaranteed to be exact/correct.

We described the most significant properties of RS tables. Our framework includes more, but it is nevertheless a fixed set of kinds for every RS table. This provides a template that can aid an analyst to generate tables, ensures consistency across different RSs, and is sufficient for an in-depth analysis of representations. See Tables 1 and 2 for some example RS tables.

\subsection{Problems}

Abstractly, a problem $q$ is a triple $\left(O_{q}, G_{q}, C_{q}\right)$ where $O_{q}$ is an initial condition, $G_{q}$ is a goal condition, and $C_{q}$ is a set of constraints on the paths leading from the states that satisfy $O_{q}$ to the states that satisfy $G_{q}$ [21].

This definition is difficult to square with the fact that problem specifications rarely look like such a triple. Our contention is that this is because most problems are about information recovery within a space determined by conventional information-manipulation rules.

For example, the Birds problem above is neither explicit about the goal condition nor about any path constraints, and we can only assume that the initial condition is whatever state things are when the problem is presented. However, a competent problemsolver will find the statement what is the probability ... informative about the type of the answer expected (specifically, ratio, percentage, or real number or any data-type usually used to encode probability values). In other words, the problem-solver is expected to perform type-inference to obtain the answer type. Moreover, the rest of the problem statement will typically provide data which, under conventional interpretations, can be manipulated to recover the answer. Thus, given a problem specification $q$, the information $\left(O_{q}, G_{q}, C_{q}\right)$ is hidden in the specification and meant to be inferred by the problem solver.

We call the set of paths that satisfy $\left(O_{q}, G_{q}, C_{q}\right)$ the semantics of the problem. The relation between a problem specification and its semantics is complex, because it requires, first, an understanding of how the specification relates to the triple (deemed problem understanding by [21]), and second, knowledge of the paths that satisfy the triple (problem solving). 
Q tables. Similar to RS tables, $\mathrm{Q}$ tables encode the properties of problems ( $Q$ properties) as kind-value pairs.

The presentation of a problem requires an RS. We write $q: S$ to denote 'a problem $q$ represented under system $S$ '. Then, a problem's properties can be encoded similar to representational systems (e.g., which types and tokens appear). The only difference is that here we qualify the properties by their semantic contribution. Thus, to build a $\mathrm{Q}$ table, an analyst is required to estimate the semantic contribution of properties. That is, they need to decide on the hierarchy of the importance of each property relative to other properties. Naturally, we do not assume full knowledge of the semantics, as this would require complete knowledge of solutions. We only assume partial understanding of the goal conditions, and any estimation of the relevance of different properties can help. See Table 3 for a $\mathrm{Q}$ table for the Birds problem.

Table 3. A Q table for the Birds problem in its natural language representation. The colour codes for importance relative to information content: essential (purple), instrumental (blue), relevant (green), circumstantial (yellow), noise (red).

\begin{tabular}{|ll|}
\hline kind & value \\
\hline error allowed & 0 \\
\hline answer type & ratio \\
\hline tokens & probability, and, not \\
\hline types & ratio, class \\
\hline patterns & _ratio of _class are_:class, probability of_:class and_:class \\
\hline facts & $\begin{array}{l}\text { Bayes' theorem, law of total probability, unit measure, additive } \\
\text { inverse, . . }\end{array}$ \\
\hline tactics & deduce, calculate \\
\hline tokens & $\begin{array}{l}\text { one, quarter, all, animals, birds, two, thirds, can, fly, half, flying, } \\
\text { X, animal, probability, cannot }\end{array}$ \\
\hline related tokens & $\begin{array}{l}\text { times, divided_by, plus, minus, equals, union, intersection, } \\
\text { probability, zero, .. . }\end{array}$ \\
\hline \# of tokens & 67 \\
\hline \# of distinct tokens & 31 \\
\hline \# of statements & 5 \\
\hline tokens & feathers \\
\hline related tokens & $\begin{array}{l}\text { beast, animate, creature, wing, aviate, flock, fowl, dame, carnal, } \\
\text { being, fauna,... }\end{array}$ \\
\hline
\end{tabular}

Properties such as error allowed and answer type are at the top of the importance hierarchy (purple/essential) because they inform us directly about the goal condition. Specifically, answer type refers to the shape of the data (data-type) expected to appear in any state satisfying the goal conditions, and error allowed refers to the rigour expected out of the answer (i.e., how permissive is the goal condition).

Next (blue/instrumental) in the hierarchy are any tokens, types, patterns, facts, or tactics with pivotal roles in the solution(s). These properties are informative about how the paths in the state space which lead to solutions look. 
One step below (green/relevant) are things which are clearly informative about the semantics of the problem, but which may also contain noise or just be informative as heuristics. This now includes tokens which may not appear in the problem specification but which may be useful along the way.

The lowest classes are either circumstances of the representation (yellow/circumstantial), or outright noise (red/noise) - that is, tokens that either appear in the specification or are evoked by it, ${ }^{3}$ which contain no information about the semantics. Notice, for example, that the Birds problem contains the statement birds have feathers which is not used by any solution. Thus, the token 'feathers' is classified as noise. Any tokens related to the zoological interpretation of the problem are taken as noise. Encoding these explicitly may be useful to understand potential missteps or distracting data in the specification.

Every Q table will have a fixed set of property kinds. This provides a template to generate tables-empirically, these proved sufficient for an effective analysis of candidate representations in relation to the problem.

\subsection{Correspondence}

We have presented a framework for encoding the properties of problems and representational systems. Now we need some method for assessing the relative value of different RS for representing a given problem. Our approach relies on the notion of correspondence, which is a way of relating Q properties with RS properties. It is the fundamental notion that we use for calculating the match of an RS to a problem.

Analogical correspondences. Different RSs are linked to each other through structurepreserving transformations. For example, an event can be encoded as a proposition, or as a set, or as a region in the plane. These relations between types form the basis of more complex analogies. For example, the conjunction of two events corresponds to the intersection of two regions, and the probability of an event corresponds to the area of its corresponding region. Furthermore, such transformations also induce the correspondence of facts. For example, if probability corresponds to area, then the Law of Total Probability corresponds to Additivity of Areas. The logic and mechanisms for reasoning through such transformations has been explored elsewhere [24]. In this work, rather than the mechanism, we are concerned with assessing the relative value that such transformations provide.

Q-specific correspondences. The analogical correspondences are induced by transformations between RSs (i.e., the mapping between tokens/types/facts/tactics). However, other problem-specific information may also be valuable for assessing RSs. For example, the error allowance of a problem informs us whether we need a rigorous RS or whether an imprecise one is sufficient (if there are other reasons for it to be valued, e.g., an approximate solution is sufficient for young children). Thus, correspondences such as between the Q property error allowed $=0$ and the RS property rigorous can be included.

\footnotetext{
${ }^{3}$ Those appearing in this example are taken from a semantic net [30].
} 
Correspondence tables. Currently, we assume that a catalogue of such transformations/analogies is known. Furthermore, we assume that we have a measure that estimates the information loss in a correspondence. We call this the correspondence strength. ${ }^{4}$ For example, the injection of natural numbers into real numbers is lossless, so the strength of type natural to type real is assumed to be 1 . However, any transformation from real to natural is lossy, so its strength must be less than 1 (the question of how much exactly is up for discussion). Thus, each correspondence can be encoded as a triple $\left(p_{q}, p_{r}, s\right)$ where $p_{q}$ and $p_{r}$ are $\mathrm{Q}$ and RS properties, respectively, and $s$ is the strength.

Each correspondence between a property of $q$ and a property of $S$ can be seen, roughly, as a reason why $q$ could be represented in $S$. Simplistically, this could mean that adding up the values of all correspondences between $q$ and $S$ might give us a score of $S$. However, the reasons may not be independent, so adding them up may count redundant correspondences multiple times. Thus, we introduce a simple calculus for specifying correspondence in the most independent possible way (e.g., see Table 4; more details are in Section 4.2).

Table 4. Some example correspondences encoded with operator OR.

\begin{tabular}{|llr|}
\hline Q property formula & RS property & strength \\
\hline type occurrence OR type class & type event & 1 \\
\hline type ratio OR type percentage & type real & 1 \\
\hline token intersection OR token and & token $\cap$ & 1 \\
\hline token given OR token if & token & 1 \\
\hline error allowed $=0$ & rigorous & 1 \\
\hline error allowed $=0$ & NOT rigorous & -1 \\
\hline
\end{tabular}

But how can Q, RS, and correspondence tables be used for representation recommendation? We present a proof-of-concept algorithm next.

\section{Using the framework}

Our tables give us properties of the problem and of the candidate RSs. Correspondence tables give us explicit links between them. The task now is to exploit this information to find correspondences which match properties of both the problem and the target RS. Not all properties bear equal importance, thus we modulate the correspondence strength. Combining these assigns a real value to each potential RS indicating its relevance as a candidate RS. Algorithm 1 implements this process.

\subsection{Matching correspondences}

In our running example, we have a Q table for the Birds problem and four RS tables for the representational systems: Bayesian, Geometric, and Contingency, and also an Euler

\footnotetext{
${ }^{4}$ In future work, we will investigate how correspondences and their strength can be identified automatically (e.g., using machine learning).
} 


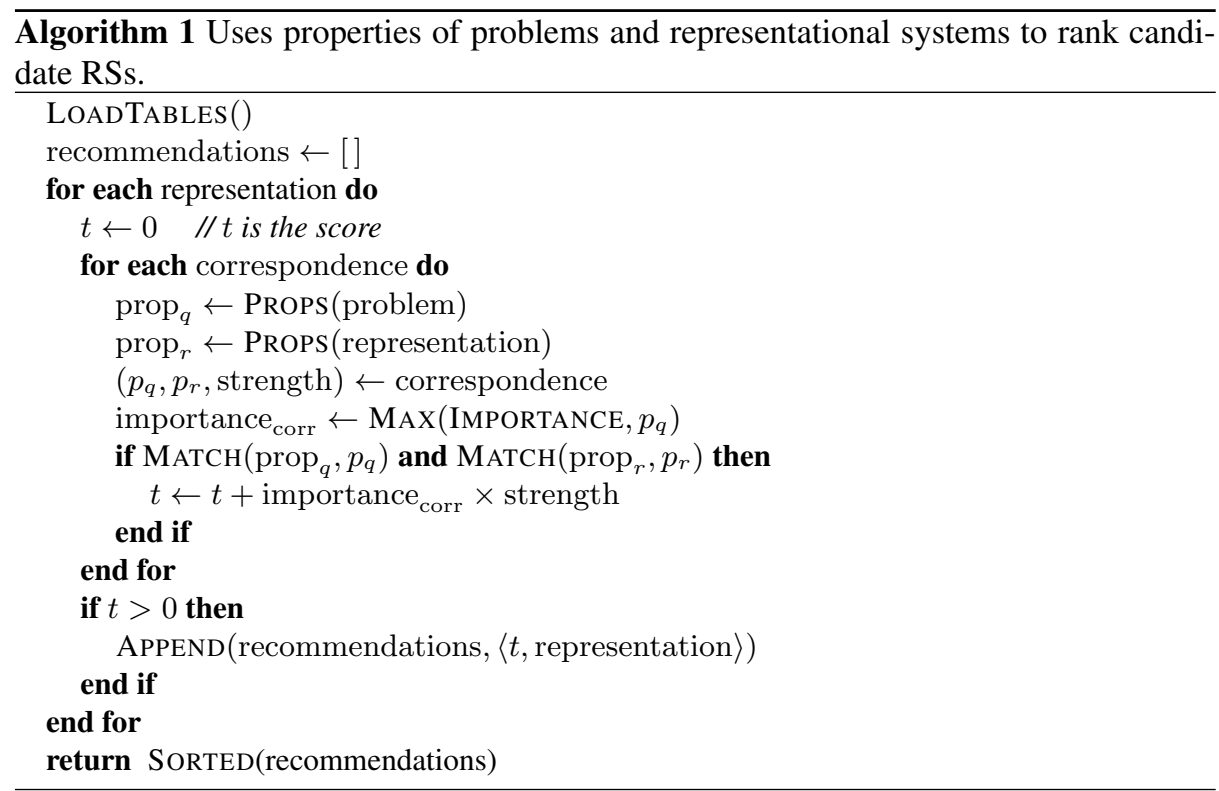

RS table. ${ }^{5}$ They are accompanied by a table of correspondences between properties. Algorithm 1 accesses these tables, and then iterates over the RSs to find correspondences linking the problem to the RS.

Suppose the first candidate representation for the Birds problem is Bayesian. Thus, we consider the Bayesian RS table and the Birds problem Q table. Next, we examine each correspondence: a triple $\left(p_{q}, p_{r}, s\right)$ where $p_{q}$ and $p_{r}$ are properties of the problem and representation, respectively, and $s \in[-1,1]$ is the correspondence strength. We examine if both Q table properties and RS table properties match the conditions of this correspondence. For example, the correspondence:

$$
\text { (error allowed }=0, \text { rigorous, } 1)
$$

from Table 4 matches properties in the Q table in Table 3 and those in the RS table from Table 1. If there is no match, we disregard this correspondence. ${ }^{6}$ When they do match — as in this example - we take the strength $s$ and modulate it by the importance of the matched Q property. Each importance colour band is assigned a value in the $[0,1]$ interval, which we multiply by the strength of the correspondence $s$. Our example correspondence involves the property error allowed, which is an essential (purple) property, so is modulated by the 'essential' value 1. Altogether, this correspondence contributes $1 \times 1=1$ to the Bayesian RS ranking score.

\footnotetext{
${ }^{5}$ By 'Euler' we mean some implementation of Euler diagrams.

${ }^{6}$ Future research includes considering the importance of question properties that remain unmatched.
} 


\subsection{Property formulae}

The $p_{q}$ and $p_{r}$ from the correspondence triple can be property formulae expressed in a simple calculus using binary connectives AND and OR, and the unary connective NOT. ${ }^{7}$ The property calculus allows for greater expressivity and better captures the nature of correspondences between Q and RS properties. We see this in the correspondence:

(type occurrence OR type class, type event, 1)

where we require one (or both) of the properties specified in the $p_{q}$ of the correspondence triple to occur in the $\mathrm{Q}$ table. In this situation, we do observe type class occurring as an instrumental (blue) property. The correspondence is matched despite the absence of type occurrence; notice that it was necessary to observe type event in the Bayesian RS table. The matched correspondence formula involved both type class and type occurrence, with the match being satisfied by an instrumental (blue) property. Thus, the correspondence strength of 1 is modulated by the 'instrumental' value 0.6 , increasing the Bayesian RS score by $1 \times 0.6=0.6$.

\subsection{Making a recommendation}

Once all correspondences for a particular Q and RS are identified and modulated by importance, we combine them to a single score. This can be done in many ways: we take a simple sum. For the example of the Bayesian representation and the Birds problem, the correspondence analysis gives an overall score of 9.3.

Repeating the scoring process above for each candidate RS yields the following recommendation ranking:

$$
\begin{array}{lr}
\text { Bayesian } & 9.3 \\
\text { Geometric } & 7.2 \\
\text { Contingency } & 5.4 \\
\text { Euler } & 1.5
\end{array}
$$

We hence recommend that the Birds problem, initially posed in a Natural Language RS, might be better attempted in the Bayesian RS. This seems a sensible recommendation.

\section{Discussion and future work}

So far we showed that representation selection can be encoded in a sufficiently formal way to computationally analyse the underlying informationally-relevant structural matches across domains. This is novel and exciting. We now evaluate the quality of performance of our framework, and discuss its significance for applications and future work.

\footnotetext{
${ }^{7}$ AND requires that both properties appear in the property table. OR requires that at least one of the properties appears in the property table. If both properties appear in the table, the strength is only counted once. NOT requires that a specified property does not occur in the property table.
} 


\subsection{Analysing the trace}

Perhaps more interesting than the resulting scores is the data that the trace of the algorithm execution provides. For example, it enables an analysis about how individual correspondences contribute to the total score. The high importance (essential) Q property answer type ratio corresponds to properties type real in the Bayesian, Geometric, and Contingency systems. However, in the Euler system it has no corresponding property. ${ }^{8}$ Similarly, the token probability corresponds to the token Pr in Bayesian, and the tactics compare sizes and compare cell values in Geometric and Contingency, respectively, but it has no correspondence in Euler. Thus, due to the high values for the importance of the essential and instrumental properties, the gap between Euler and the other RSs widens. This is expected and desirable. ${ }^{9}$

We observe that tokens in some systems (e.g., probability) can correspond to tactics (e.g., compare sizes) without corresponding to any specific tokens. This is interesting from the cognitive perspective, because these tokens and tactics are very different operationally. A cognitively focused analysis may be able to assess the impact of differences of this sort.

The trace analysis has many potential applications. We envision tutoring systems that can make specific recommendations (e.g., "maybe you can draw a region in space to represent the class of animals"), or explainable AI systems that can justify their decisions in a humanly-understandable manner.

Zero-weight properties. Some Q and RS properties make no contribution in terms of correspondence scores, for example, all the circumstantial and noise properties. Nevertheless, we chose to encode them in our framework because they have potentially important effects on cognitive processes. For example, the total number of (circumstantial) tokens may be used to estimate the cognitive cost of registering a problem specification. Moreover, the inference power of an RS may make it more applicable from a formal point of view, but the cost of using it may be higher from a cognitive point of view.

An analysis of the correspondence between noise properties of Q and RS may be used for predicting human error or bias. For example, a novice user might be tempted to represent zoological facts given in the Birds problem, but they contribute to unnecessary cognitive costs.

\subsection{Evaluating the framework and algorithm}

To our knowledge, no work has been done on computationally modelling representation selection, so we have no benchmarks by which to judge our framework or algorithm. The execution of the algorithm presented here is a proof-of-concept application, but it shows that given our encoding of a problem $Q$ and various representations RSs, we can

\footnotetext{
${ }^{8}$ In Euler diagrams the cardinality of sets is abstracted away; the size of zones is meaningless.

${ }^{9}$ It is also noteworthy that the strength of the correspondences from probability to Pr and compare sizes was set to 1 (because in principle any probability function is representable in the Bayesian or Geometric systems), but it was set to 0.5 for Contingency because not every probability function is representable in Contingency tables.
} 
compute interesting measures. Moreover, the approach that we take for encoding the description of problems, RSs and correspondences makes minimal assumptions about them. This makes it general. So far, we have encoded 9 different RSs and various problems.

One of the main limitations of our framework is the need for an analyst to encode the properties of Qs and RSs, the correspondence strengths, and the importance that each Q property has relative to potential solutions. This clearly requires the analyst to understand the complexity of a problem, and to have at least some understanding of how a solution would look (e.g., identifying potentially instrumental facts). This poses a problem for automation. One way of tackling this is with the help of machine learning methods similar to the work of [18] for lemma selection.

\subsection{Future work}

Our framework opens up many avenues for future research. Automating the generation of Q and RS tables and their importance is a clear goal to be achieved. We are currently including methods for analysing the cognitive properties of representations, and want to extend the framework to include user profiles next. We are curious to find out if representation selection based on our framework can promote problem solving or learning in humans, and want to incorporate it into a personalised multi-representation tutoring system.

\section{Conclusion}

We have presented a novel framework for computationally selecting suitable representations. We introduced the language, data structures, and methods for encoding and analysing the properties of problems, of representational systems, and the correspondences between them.

Our proof-of-concept algorithm ranks representational systems according to a measure of suitability given a problem. The algorithm analyses the problem's properties in terms of their informational contribution and estimates the likelihood that the problem's semantics can be recovered in each candidate RS. We see this work as an exciting foundation upon which we can build the machinery to analyse cognitive properties, so the user profile may be included to calculate a recommendation. 


\section{Bibliography}

[1] Shaaron Ainsworth. The functions of multiple representations. Computers \& education, 33(2-3):131-152, 1999.

[2] Dave Barker-Plummer, John Etchemendy, Albert Liu, Michael Murray, and Nik Swoboda. Openproof-a flexible framework for heterogeneous reasoning. In International Conference on Theory and Application of Diagrams, pages 347-349. Springer, 2008.

[3] Yoshua Bengio, Aaron Courville, and Pascal Vincent. Representation learning: A review and new perspectives. IEEE transactions on pattern analysis and machine intelligence, 35(8):1798-1828, 2013.

[4] Alan Blackwell and Thomas Green. Notational systems-the cognitive dimensions of notations framework. HCI models, theories, and frameworks: toward an interdisciplinary science. Morgan Kaufmann, 2003.

[5] Peter C-H Cheng. Unlocking conceptual learning in mathematics and science with effective representational systems. Computers \& Education, 33(2-3):109130, 1999.

[6] Peter C-H Cheng. Probably good diagrams for learning: representational epistemic recodification of probability theory. Topics in Cognitive Science, 3(3):475498, 2011.

[7] Peter CH Cheng. What constitutes an effective representation? In International Conference on Theory and Application of Diagrams, pages 17-31. Springer, 2016.

[8] Noam Chomsky. Three models for the description of language. IRE Transactions on information theory, 2(3):113-124, 1956.

[9] Noam Chomsky. On certain formal properties of grammars. Information and control, 2(2):137-167, 1959.

[10] Thierry Coquand. Type theory. 2006.

[11] Details anonymised.

[12] John Harrison. HOL light: An overview. In International Conference on Theorem Proving in Higher Order Logics, pages 60-66. Springer, 2009.

[13] Brian Huffman and Ondřej Kunčar. Lifting and transfer: A modular design for quotients in isabelle/hol. In International Conference on Certified Programs and Proofs, pages 131-146. Springer, 2013.

[14] Mateja Jamnik, Alan Bundy, and Ian Green. On automating diagrammatic proofs of arithmetic arguments. Journal of logic, language and information, 8(3):297$321,1999$.

[15] Jupyter. jupyter.org.

[16] Matt Kaufmann and J Strother Moore. Acl2: An industrial strength version of nqthm. In Proceedings of 11 th Annual Conference on Computer Assurance. COMPASS'96, pages 23-34. IEEE, 1996.

[17] Laura Kovács and Andrei Voronkov. First-order theorem proving and vampire. In International Conference on Computer Aided Verification, pages 1-35. Springer, 2013. 
[18] Daniel Kühlwein, Jasmin Christian Blanchette, Cezary Kaliszyk, and Josef Urban. Mash: machine learning for sledgehammer. In International Conference on Interactive Theorem Proving, pages 35-50. Springer, 2013.

[19] Matlab. mathworks.com.

[20] Till Mossakowski, Christian Maeder, and Klaus Lüttich. The heterogeneous tool set, hets. In International Conference on Tools and Algorithms for the Construction and Analysis of Systems, pages 519-522. Springer, 2007.

[21] Allen Newell. Human Problem Solving. Prentice-Hall, Inc., 1972.

[22] Tobias Nipkow, Lawrence C Paulson, and Markus Wenzel. Isabelle/HOL: a proof assistant for higher-order logic, volume 2283. Springer Science \& Business Media, 2002.

[23] GNU Octave. octave.org.

[24] Daniel Raggi, Alan Bundy, Gudmund Grov, and Alison Pease. Automating change of representation for proofs in discrete mathematics (extended version). Mathematics in Computer Science, 10(4):429-457, 2016.

[25] SageMath. sagemath.org.

[26] Gem Stapleton, Mateja Jamnik, and Atsushi Shimojima. What makes an effective representation of information: a formal account of observational advantages. Journal of Logic, Language and Information, 26(2):143-177, 2017.

[27] M. Urbas and M. Jamnik. A framework for heterogeneous reasoning in formal and informal domains. In T. Dwyer, H.C. Purchase, and A. Delaney, editors, Diagrams, volume 8578 of Lecture Notes in Computer Science, pages 277-292. Springer, 2014.

[28] Matej Urbas, Mateja Jamnik, Gem Stapleton, and Jean Flower. Speedith: a diagrammatic reasoner for spider diagrams. In International Conference on Theory and Application of Diagrams, pages 163-177. Springer, 2012.

[29] Daniel Winterstein, Alan Bundy, and Corin Gurr. Dr. doodle: A diagrammatic theorem prover. In International Joint Conference on Automated Reasoning, pages 331-335. Springer, 2004.

[30] WordNet. wordnet.princeton.edu, 2010. 